\title{
Long-term variations of the flow direction and angular momentum of the solar wind observed by Helios
}

\author{
K. Scherer ${ }^{1}$, E. Marsch ${ }^{2}$, R. Schwenn ${ }^{2}$, and H. Rosenbauer ${ }^{2}$ \\ 1 dat-hex, Obere Straße 11, 37191 Katlenburg-Lindau, Germany \\ 2 Max-Planck Institut für Aeronomie, 37191 Katlenburg-Lindau, Germany
}

Received 18 May 1999 / Accepted 8 November 2000

\begin{abstract}
The flow directions of solar wind protons were measured in situ by the Helios spacecraft. A long-term average of the velocity shows a systematic drift in the latitudinal flow angle of about $+1^{\circ}$ north observed with Helios 1 and $-1^{\circ}$ south observed onboard of Helios 2. The longitudinal flow angle migrates about $+1^{\circ}$ west over a period of almost 10 years for Helios 1 and 6 years for Helios 2. This systematic change with time of the plasma flow direction may be caused by solar-cycle variations of the orientation of the Sun's magnetic field which partially corotates with the Sun inside the Alfvén surface (varying in distance between $10 R_{\odot}$ over the poles and $30 R_{\odot}$ near the equator). These variations must have been imprinted on the solar wind flow when it detached from corotation with the Sun near the Alfvén point. The angular momentum of the wind is intimately connected with the flow and field directions. The gain of total angular momentum of the wind equals the loss of angular momentum of the Sun, which is caused by the torque exerted on the rotating Sun through the magnetic field of the expanding corona. Implications of the Helios observations for models of the magnetic fields of the Sun as well as the solar wind are discussed. We show evidence, that changes of the solar magnetic field inside the Alfvén surface are responsible for systematic drifts in the solar wind flow direction.
\end{abstract}

Key words. Sun: solar wind - Sun: magnetic fields - Sun: activity

\section{Introduction}

The distant solar wind is observed to expand nearly radially outward from the Sun, such as assumed in the early models (e.g. Parker 1963). However, close to the Sun the flow is non-radial and strongly guided by the magnetic field. Only beyond the Alfvén point is the plasma flow no longer governed by the Sun's magnetic field, and from thereon the field is frozen in the plasma and carried away by the solar wind (Weber \& Davis 1967). Due to solar rotation the interplanetary magnetic field in the ecliptic plane is wound into a spiral. The global heliospheric field has the simplest form during the minimum phase of the solar cycle (for a review see e.g. Forsyth et al. 1996). The solar minimum field configuration has recently been modeled analytically by Banaszkiewicz et al. (1998). Their model consists of a dominant dipole and a weaker quadrupole component plus a field caused by the near-Sun current sheet. This sheet extends radially outward and transforms gradually into the heliospheric current sheet.

Because in a heliocentric ecliptic coordinate system (used throughout this paper) neither the magnetic equator

Send offprint requests to: K. Scherer,

e-mail: dat-hex@t-online.de is aligned with the ecliptic plane nor the solar rotation axis with the polar axis, the current sheet depends on the ecliptic latitude. Observationally, the current sheet is warped (Hoeksema et al. 1982, 1983) and thus causes an additional latitudinal dependence ("ballerina skirt") of the magnetic field. The near-Sun wind, i.e., the wind between the source surface of the magnetic field at about $3 R_{\odot}$ (Altschuler \& Newkirk 1969; Schatten et al. 1969; Hoeksema et al. 1982, $1983)$ and the Alfvén surface $<30 R_{\odot}$ according to Marsch \& Richter (1984), is guided by the coronal magnetic field, which has no toroidal (azimuthal) component in a corotating frame. Therefore, the near-Sun wind will, while being guided by the magnetic field, acquire sizeable non-radial velocity components in a non-corotating heliocentric rest frame. These components remain and are imprinted on the solar wind while it passes through the Alfvén point.

In the above picture the global heliospheric magnetic field is divided into three separate components: the almost current-free field inside the source surface about $3 R_{\odot}$, the current driven heliospheric magnetic field (HMF) outside the Alfvén surface $\left(<\approx 30 R_{\odot}\right)$, and the intermediate field in between. The latter acts as a kind of magnetic clutch, between the coronal magnetic field and the HMF. In the region of the intermediate field also the 
acceleration of the slow solar wind occurs, and thus this region is dynamically very important. Nevertheless, this picture is oversimplified, because at high latitudes $\left(>\left| \pm 40^{\circ}\right|\right)$ the Alfvén surface may intersect the source surface, especially in the polar regions. Therefore, the three-region model may be applied best at low latitudes, where the Helios spacecraft were located.

In the interplanetary space, the non-radial components of the solar wind velocity are usually less than $10 \%$ of the radial component. The wind speed, $V_{\mathrm{sw}}$, is varying between 250 and $800 \mathrm{kms}^{-1}$ (see e.g. the Helios solar wind speed statistics in Schwenn 1990). Recently, interest arose again to study non-radial flows with data obtained in the outer heliosphere. Richardson et al. (1995) discussed the longitudinal variation derived from the data of the Pioneer Venus Orbiter (PVO), the Interplanetary Monitoring Platform (IMP 8), WIND, and Voyager 2. The data obtained from these spacecraft extend over many years and cover the heliocentric distance range from $0.7 \mathrm{AU}$ to $45 \mathrm{AU}$. The combined data set can thus be used to study the radial, longitudinal and, within limits, even latitudinal variations of the wind flow angles and a possible dependence on the solar cycle. Similarly, the Helios data can be used to study the non-radial flows at distances between $0.3 \mathrm{AU}$ and 1.0 AU and their long-term trends in connection with the Sun's magnetic field. Such a study is the main aim of the present paper. The angular momentum of the solar wind measured within Earth's orbit has been analyzed earlier by Pizzo et al. (1983) and Marsch \& Richter (1984), who also inferred the location of the Alfvén critical point, $R_{\mathrm{A}}$. Assuming that the specific angular momentum, $L=\Omega_{\odot} R_{\mathrm{A}}^{2}$, is conserved allows one to determine $R_{\mathrm{A}}$, which can be inferred from the measured $L$ value and is found to range between about 10 and $30 R_{\odot}$. Here $\Omega_{\odot}$ is the solar rotation frequency.

However, temporal and spatial variations of the boundary conditions in the solar corona as well as interplanetary plasma kinematics and dynamics can change the flow direction of the expanding wind, and thus an interpretation of the azimuthal (east-west) component of the solar wind must consider two effects, which have been known (see, e.g., Dessler 1967; Pizzo et al. 1983) for a long time:

1. The coronal plasma corotates partly with the Sun up to about the Alfvén point, beyond which the plasma is gradually released from corotation. Thus the rotating Sun enforces on the wind an azimuthal (or latitudinal) flow component, which is added on the dominant radial velocity component. If the global coronal magnetic field changes with time or in space the non-radial velocity components in the wind will change accordingly;

2. When a fast solar wind stream, whose field lines are less bent, collides with a preceding slow stream, which has a stronger spiral curvature, then compressive waves are excited without plasma interpenetration. As a result, an eastward (westward) deflection of the fast (slow) solar wind is produced and seen by a stationary in-situ observer crossing the interaction region as a perturbation of the flow from the radial direction.

The first effect has the following consequence, resulting from conservation of the mechanical angular momentum: for any unperturbed fluid parcel, $v_{\perp 0} r_{0}=v_{\perp} r$, where $v_{\perp_{0}}$ is the transverse (to the radial) velocity component at $r_{0}=R_{\mathrm{A}}$, where the corotation ceases, and $v_{\perp}$ is the velocity at a larger distance, $r$, and decreases inversely proportional to the radius. Thus if the reference value $v_{\perp 0}$ and release height $r_{0}$ in the corona change, the interplanetary flow direction of the wind must change as well. The solar corotation velocity at the equator of the Sun is $2 \mathrm{~km} \mathrm{~s}^{-1}$. At $1 \mathrm{AU}$ one still observes on Helios values of the transverse wind velocity, $v_{\perp}$, in the range from $\approx 1, \ldots 9 \mathrm{~km} \mathrm{~s}^{-1}$. Such large azimuthal components were first reported by Brandt (1966). Observationally, the slow wind near the ecliptic plane has the largest remnant azimuthal velocity component. If extrapolated back to the Sun, these values correspond to corotation distances of $r_{0} \approx 14 \ldots 30 R_{\odot}$ according to the Helios observations and the Weber \& Davies (1967) model. The distance $R_{\mathrm{A}}$ is the point where the solar wind speed exceeds that of Alfvén waves in the outer corona, or from the dynamical point of view, where the wind ram pressure exceeds the energy density of the Sun's magnetic field.

It should be noted that the angular momentum of a solar wind parcel not only depends on the distance at which it is released, but also on its heliospheric latitude at which it is released from corotation. At the poles the latitudinal (azimuthal) component vanishes identically. A natural choice of coordinates would therefore be the heliographic coordinates instead of the ecliptic spacecraft coordinate system. On the other hand, the angular momentum is an invariant, and therefore independent of the coordinate system. For this reason, we have used the original data in the ecliptic spacecraft coordinate system.

The second effect discussed above will cause compressions and deflections by stream interactions (corotating interaction regions, "CIR"), which can result in forward and reverse shock waves (see Gosling et al. 1997, for a recent review of the Ulysses observations). In addition to the deviations of the solar wind from the radial direction induced by its corotation up to the Alfvén surface, CIRs produce deviations of the solar wind from the radial direction. Such deflections are easily detectable when the wind passes over a stationary observer. Because a pair of shock waves is usually created (with a contact discontinuity in between), the first shock wave causes an eastward drift and the second one a westward drift (Dessler 1967). Also, Ulysses made some good observations of the north-south flow deflections associated with CIRs, which are discussed by Gosling et al. (1997) and in earlier papers by the same author. Because CIRs are mostly emerging only beyond $0.63 \mathrm{AU}$ (e.g. Schwenn 1990), and since the opposite drifts almost cancel on a long-term average, this effect will not be discussed in the following. Stream collisions become increasingly important in the outer 
heliosphere (e.g., Burlaga 1995), but their effects are less important in the orbital range of Helios where the interaction regions are still developing. Also, meridional (northsouth) components may arise, which are thought to be driven by stream collisions and associated pressure gradients (Richardson et al. 1995; Gosling et al. 1997). In this paper we will present evidence supporting the argument that the changes of the magnetic field inside $R_{\mathrm{A}}$ are responsible for the observed mean systematic drifts in the plasma flow direction.

\section{Data analysis}

\subsection{The solar wind flow direction}

The twin Helios spacecraft were virtually identical and both placed in highly-eccentric heliocentric orbits, having perihelia at about $0.3 \mathrm{AU}$ and aphelia at about $1 \mathrm{AU}$. To facilitate an accurate determination of the angular momentum flux of the solar wind, the attitudes of Helios 1 and Helios 2 and thus the spin directions were opposite to each other, with Helios 1 spinning in the same sense as the Sun. In the plasma instrument a set of contiguous electron multipliers (CEM) sorts the particles according to the direction of incidence in elevation with respect to the ecliptic. Nine CEMs provide this directional resolution in the spacecraft polar angle $(\vartheta)$, while the azimuthal $(\varphi)$ angular resolution is obtained by using the spin of the spacecraft. A more detailed description can be found in Pizzo et al. (1983), who also discuss the subtleties involved in a reliable measurement of the azimuthal flow angle and the angular momentum. In the present $1-\mathrm{D}$ analysis their results have been taken into account, and our data have been corrected in the same way as discussed by Pizzo et al. (1983). In the above publication, the authors show that the error in the azimuthal velocity is less than $1 \mathrm{~km} \mathrm{~s}^{-1}$ at $1 \mathrm{AU}$. Therefore, our results are at the limit of the velocity resolution of the plasma instruments on the Helios spacecraft. Because of the long-term averages the presented data will at least show the quantitative trend. Yet, because of the small angles involved, we cannot eliminate for certain that the systematic drift is not partly due to an instrumental or spacecraft effect.

In our present analysis we used a data set of dailyaveraged parameters. The data were further smoothed by a running mean over a period of 350 days. The trend is also clearly seen in shorter time averages ( $>30$ days), but there are still short period variations due to the orbit of the spacecraft. The averaging time (350 days) is quite large but appropriate, since it allows one to study the secular variation of the parameters in the data set. The averaging period is chosen in such a way that the data become smooth, but it does not coincide and thus is not in conflict with multiples of other known periodicities, such as 1 year, the $\approx 180$ days orbital revolution period of the spacecraft, or the 25.3 days sidereal rotation period of the Sun. With the average parameters we can analyze the

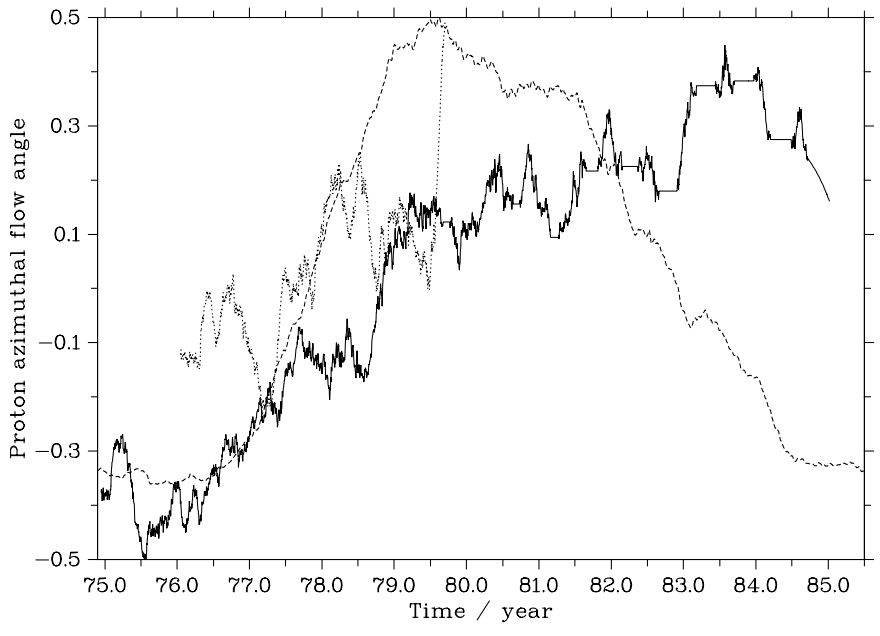

Fig. 1. A 350-days running mean of the azimuthal flow angle (in degrees) of the solar wind: Helios 1 (solid line), Helios 2 (dotted line) and the daily sunspot number (dashed line) as an indicator of the phase of the solar cycle. The data are the deviations of the flow directions from the overall average, which is $\alpha=-1.60^{\circ}$ for Helios 1 and $\alpha=2.78^{\circ}$ for Helios 2

long-term trends of the flow angles and study their dependence on time or on the solar cycle.

The results for the azimuthal (longitudinal) flow angle are shown in Fig. 1. It can be seen from Fig. 1 that the average azimuthal flow direction of the solar wind changes continuously and has a systematic eastward drift measured at both spacecraft with a mean rate of $0.1^{\circ}$ year, which where estimated by a linear least square fit. The solid line represents the Helios 1 data, the dotted the Helios 2 data. For comparison the averaged sunspot number (dashed line) is also shown. There seems to be no correlation of the flow-angle drift with the solar cycle. The horizontal lines in Fig. 1 and the following figures are caused by data gaps. For some periods at the the end of the missions data were only collected during the perihelion passage. During that time there was no data coverage at the beginning and end of the 350 day average causing the straight lines in Figs. 1 and 2.

The results for the elevation (latitudinal) flow angle are shown in Fig. 2. It can be seen from Fig. 2 that the average elevation of the flow of the solar wind changes continuously and has a systematic drift from north to south or south to north, respectively, which is measured at both spacecraft with a mean rate of about $0.2^{\circ} /$ year. These latitudinal drifts show a different behavior in the Helios 1 and Helios 2 data: both drifts seem to be anti-correlated or perhaps strongly phase-shifted in time by about 2.5 to 3 years. The Helios 1 data appear to lead the solar cycle (indicated by the sunspot number given by the dashed line), while the Helios 2 data are most probably anti-correlated to the solar cycle variations. Yet, the data coverage is not sufficient to perform a conclusive correlation analysis.

On both spacecraft, there are identical instruments, but the spin axis of Helios 1 and 2 were inverted at launch. Therefore, one would expect that any systematic drift 


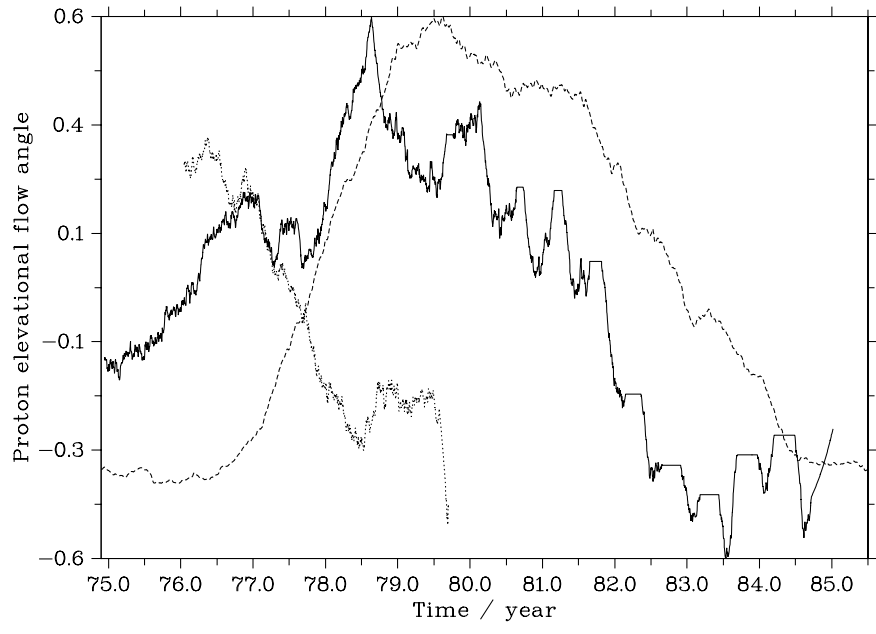

Fig. 2. A 350-days running mean of the elevation flow angle (in degrees) of the solar wind: Helios 1 (solid line), Helios 2 (dotted line) and the daily sunspot number (dashed line) as an indicator of the phase of the solar cycle. The data are the deviations of the flow directions from the overall average, which is $\epsilon=-0.79^{\circ}$ for Helios 1 and $\epsilon=-0.808^{\circ}$ for Helios 2. Note the distinct time lag between the two curves

caused by the instrument should produce equal, but opposite biases. While this may be approximately true in the latitudinal component, as shown in Fig. 2, it does not hold true for the azimuthal variation shown in Fig. 1, where both westward drifts do not show an opposite behavior. Moreover, the latitudinal effect seems to be stronger on Helios 1 than on Helios 2 (cf. Fig. 2). Therefore, we conclude that these small effects are real and not caused by any instrumental bias. Pizzo et al. (1983) have already mentioned that there might exist a difference between the angular momentum fluxes measured on Helios 1 and 2 . Obviously, their data analysis is much more detailed, because they use a $3-\mathrm{D}$ analysis, while we use the $1-\mathrm{D}$ results. On the other hand, while the results of Pizzo et al. (1983) were restricted to the Carrington rotations 1621 to 1625 (13 December 1974 to 6 April 1975), our analysis covers the entire mission, approximately 10 years for Helios 1 and about 6 years for Helios 2. To corroborate our results a $3-\mathrm{D}$ analysis over the entire mission is required, which is not available but shall be presented in a forthcoming publication.

A cross-correlation analysis was performed to estimate the lag between the time profiles of the sunspot number and the elevation flow angle as measured by Helios 1 . The daily sunspot numbers were averaged in the same way as the Helios data, and then a cross-correlation analysis (e.g. Scherer et al. 1997) was performed with the help of the Fast Fourier Transform (FFT) technique. Unfortunately, the data set does not cover a complete solar cycle. Moreover, the solar cycle 21 started in mid 1976, almost 1.5 years later than the start of the Helios data acquisition, and ceased at the end of 1986. Thus the Helios 1 data were collected during the last 1.6 years in cycle 20 (with a length of 11.6 years) and for about 9 years in

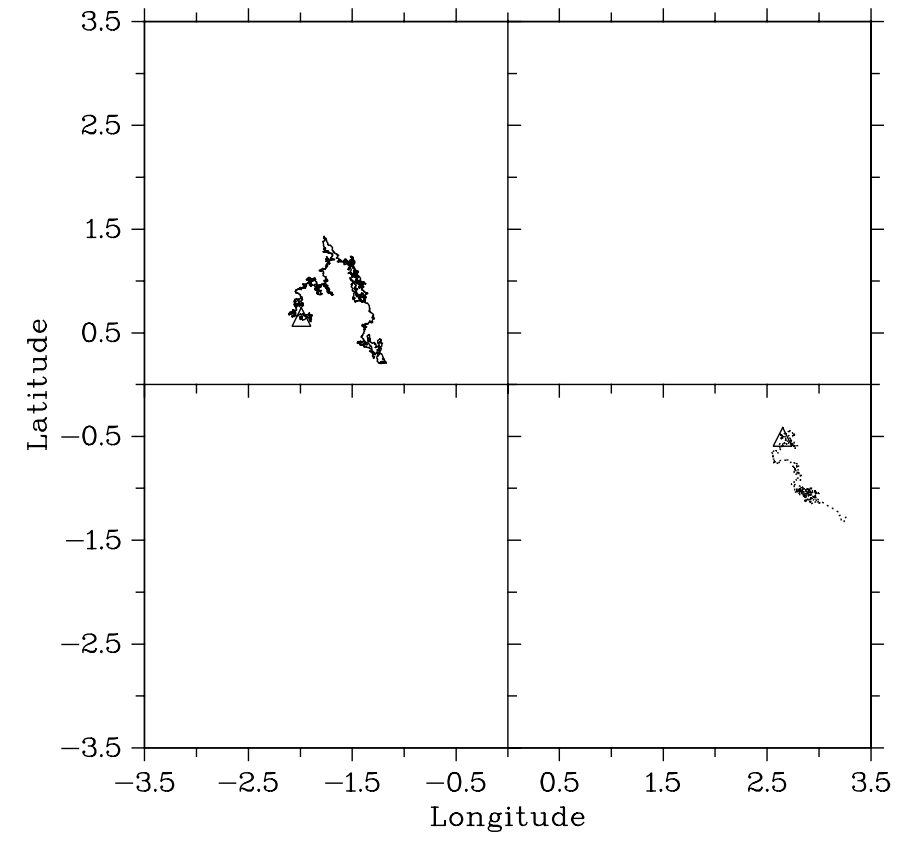

Fig. 3. Hodogram of the longitude versus latitude of the plasma flow direction, where the upper panel panel shows Helios 1 data, and the lower right panel Helios 2 data

cycle 21 (with a length of 10.3 years). Hence our data set covers about $13 \%$ of cycle 20 and and $87 \%$ of cycle 21 , and therefore a FFT-decomposition contains most probably superpositions of two or more higher-order harmonics of the solar cycle 20 and 21. Therefore, an additional cross-correlation analysis was done starting at solar cycle 21. The results from the two cross-correlation analyzes are systematically different, but show a common time lag of approximately one to two years. Therefore, a quantitative analysis of the data cannot be made. It can only be stated, that variations in the elevation flow angle measured on Helios 1 are correlated with the solar cycle with a phase shift of the order of one year. The changes of the elevation flow angle observed on Helios 2 seem to be anti-correlated with that of Helios 1, and hence with the sunspot number, but the data coverage is too small to allow a quantitative analysis. There are more advanced methods to analyse time series, which will be applied after a 3-D analysis of the data has been performed.

The long-term non-radial directional variations of the solar wind velocity with time are presented in the form of a hodogram in Fig. 3 (triangles denote the start points), where the latitude is plotted versus the longitude for the data sets of Helios 1, falling in the upper left box, and for Helios 2, falling in the lower right box. It can be seen that the Helios 1 data form roughly a ragged circle, indicating a partial rotation of the flow direction about its mean value during a full solar cycle. The data set of Helios 2 is too short to reveal a similar behavior. A qualitative interpretation of these observations will be given below. 


\subsection{The variation of the solar wind angular momentum}

In this section we provide a short analysis of the solar wind mechanical and magnetic angular momentum. For the Helios data, these quantities have been analyzed before in considerable detail by Pizzo et al. (1983) and Marsch \& Richter (1984), but for limited time periods. Here the emphasis will be on long-term trends during the solar cycle. It is easy to calculate, with the help of the plasma flow velocity and magnetic field vector, the total specific angular momentum, $\boldsymbol{L}=\boldsymbol{L}_{\mathrm{P}}+\boldsymbol{L}_{\mathrm{M}}$, per unit mass density, and its variation with the solar wind flow. Let us for the moment assume spherical symmetry and co-alignment of the rotational and magnetic axes, and that this axis is perpendicular to the ecliptic plane, which is a common assumption in most solar corona and wind models (such as Weber \& Davis 1967). By definition, the specific mechanical angular momentum is $\boldsymbol{L}_{\mathrm{P}}=\boldsymbol{r} \times \boldsymbol{u}$, i.e. the radial part vanishes identically. The other two components are obtained from the longitudinal and latitudinal components of the solar wind bulk velocity, $\boldsymbol{u}$, i.e. we have $L_{\mathrm{P} ; \varphi, \vartheta}=r u_{\varphi, \vartheta}$. Note, that the observed angular momentum vector is described in the heliocentric ecliptic polar coordinate system, and that it may generally have two components for non-radial flows (by definition, the radial component of $\boldsymbol{L}_{\mathrm{P}}$ is zero). In addition, we have calculated the components of the angular momentum, $L_{\mathrm{M}}$, of the magnetic field (Marsch \& Richter 1984) according to the formula:

$L_{\mathrm{M} ; \varphi, \vartheta}=-r \frac{B_{\varphi, \vartheta} B_{\mathrm{r}}}{4 \pi \rho u_{\mathrm{r}}}$

Here $r$ denotes the radial distance from the Sun, $B_{\mathrm{r}}, B_{\varphi}, B_{\vartheta}$ are the magnetic field components in radial, azimuthal and elevation direction in heliocentric polar coordinates, $u_{\mathrm{r}}$ is the radial component of the solar wind bulk (protons only) velocity, and $\rho=n_{\alpha} m_{\alpha}+n_{\mathrm{p}} m_{\mathrm{p}}$ is the total mass density of the $\alpha$-particles and protons, with the respective number densities $n_{i}$ and masses $m_{i}(i \in(\alpha, p))$. The latitudinal component would vanish in the ecliptic for a rotationally symmetric solar magnetic field according to the Weber \& Davis (1967) model.

Note, however, that the Sun's rotation axis is tilted by $7.25^{\circ}$ with respect to the ecliptic plane and the magnetic pole does not coincide with the rotation axis. Since the coronal field is more symmetric about the magnetic axis rather than the rotation axis, it is not just the tilt of the rotation axis that may be important. Consequently, the interplanetary magnetic field evolving from such a coronal field will naturally have a non-vanishing latitudinal component as well. Therefore, the total angular momentum, measured in a heliocentric ecliptic coordinate system will have latitudinal and longitudinal components. The spacecraft system is a Cartesian coordinate system, in which the $x$-axis is aligned with the Sun-spacecraft axis. The $z$-component is perpendicular to the ecliptic plane and the $y$-component completes the right-handed
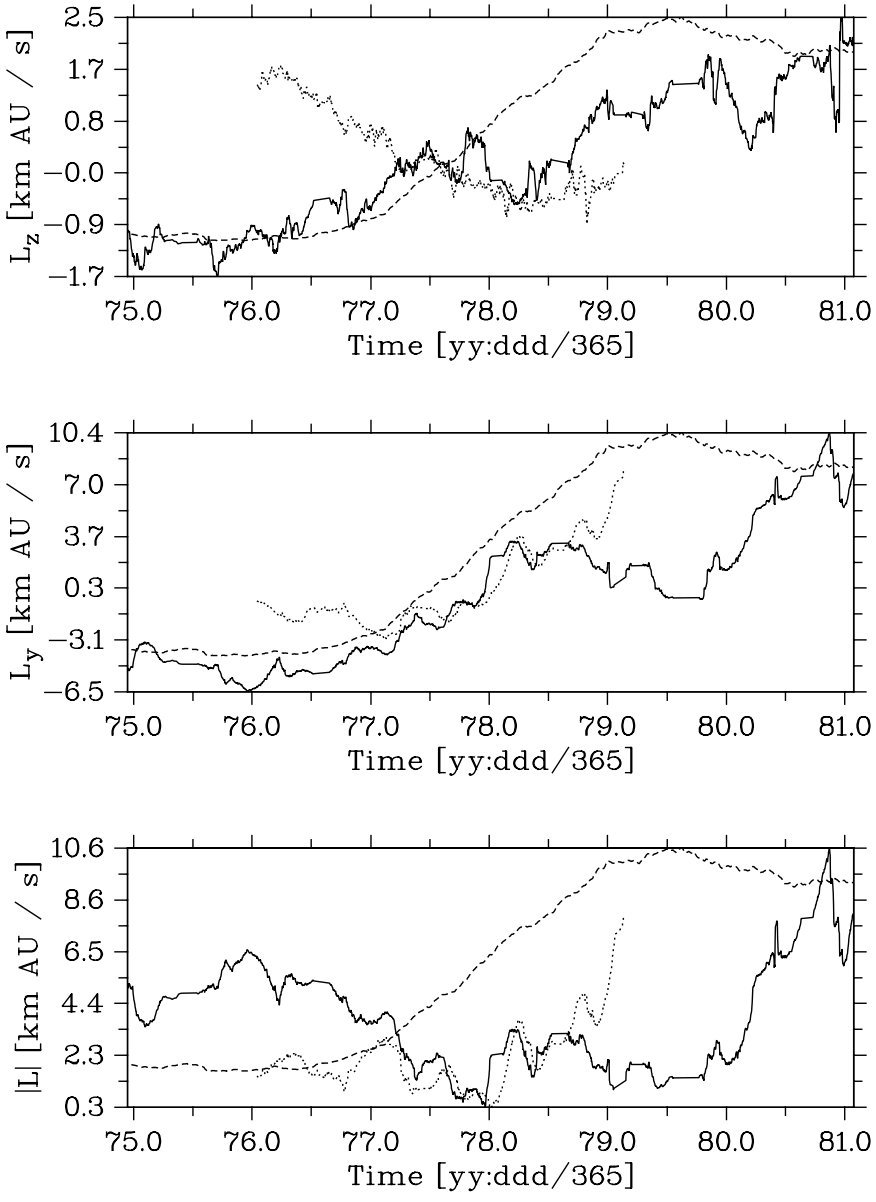

Fig. 4. Total angular momentum of the solar wind: the upper panel shows the latitudinal and the middle panel the longitudinal component of the specific total angular momentum. The bottom panel shows $L$. The solid line refers to Helios 1 and the dashed line to Helios 2. The sunspot number (dashed line) is shown for comparison

system. The spacecraft coordinate system will be used in the following.

In the upper two panels of Fig. 4, the variations of the two components, $L_{y, z}$, of the total angular momentum, $\boldsymbol{L}=\boldsymbol{L}_{\mathrm{P}}+\boldsymbol{L}_{\mathrm{M}}$ are shown. The middle panel shows the change with time of the azimuthal component, $L_{y}$, which seems, for both spacecraft, to be grossly correlated with the sunspot number. The mean azimuthal angular momentum is $L_{y}=0.135 \mathrm{AU} \mathrm{kms}^{-1}$ for Helios 1 and $L_{y}=0.103 \mathrm{AU} \mathrm{km} \mathrm{s}^{-1}$ for Helios 2.

The relative variations of the latitudinal components of the total angular momentum (upper panel of Fig. 4) are significant and appear to be anti-correlated. The mean latitudinal momentum is $L_{z}=-0.001 \mathrm{AU} \mathrm{km} \mathrm{s}^{-1}$ for Helios 1 and $L_{z}=0.247 \mathrm{AU} \mathrm{km} \mathrm{s}^{-1}$ for Helios 2. Our results indicate clearly that also the signs of the latitudinal solar wind angular momenta are different for the two spacecraft. This difference may be connected with the different heliographic positions of the spacecraft. Our results are obtained from data that are averaged over almost two orbital periods, and hence reveal the fictitious spacecraft 
The heliocentric orbits of the Helios spacecraft and the inner planets

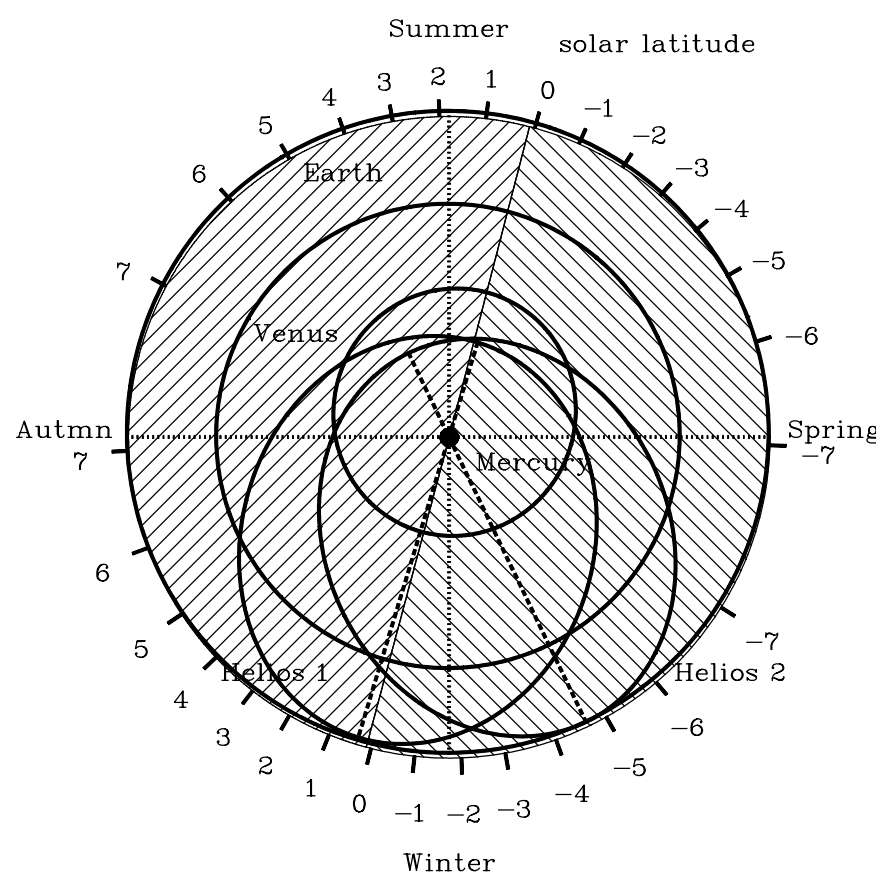

Fig. 5. The orbits of the twin Helios spacecraft and terrestrial planets. The numbers describe the projection of the heliographic latitude on the ecliptic. The shaded areas correspond to the northern and southern hemispheres, respectively

position averaged over one full Helios orbit, which is determined by: $-\frac{3}{2} a \epsilon \boldsymbol{P}$ (Scherer 2000), where $a \approx 0.64 \mathrm{AU}$ is the semimajor axis, $\epsilon \approx 0.53$ the eccentricity, and $\boldsymbol{P}$ the unit vector pointing to the perihelion. On average, both spacecraft are at a radial distance of $0.5 \mathrm{AU}$. But while Helios 1 is at $0.5^{\circ}$ in the northern solar hemisphere, Helios 2 stays on average at $5^{\circ}$ in the southern solar hemisphere. This sizable difference in latitude, which is illustrated in Fig. 5, has the consequence that the two spacecraft see different interplanetary fields and are magnetically connected to different regions in the solar corona. In the bottom panel of Fig. 4 the modulus of $\boldsymbol{L}$ is shown, indicating similar temporal variations of the Helios 1 and 2 data for about 2 years out of 3 years of available Helios 2 data. There is a anticorrelation to the solar cycle, of the specific angular momentum as measured on the Helios 1 spacecraft. But a more advanced analysis and higher data resolution are required to confirm this result.

\section{Interpretation}

In order to change the average flow direction of the interplanetary solar wind and the corresponding angular momenta, a global systematic variation of the Sun's rotation axis or magnetic field axis is required. We will first examine possible gravitational and then magnetic effects.

\subsection{Gravitational effects}

The rotation axis of the Sun may undergo a free precession. Assuming that the Sun is a homogeneous sphere, the precession frequency, $\Omega_{\mathrm{p}}$, can be estimated:

$\Omega_{\mathrm{p}}=\frac{C-A}{C} \Omega_{\odot}$

where $\Omega_{\odot}=\frac{2 \pi}{T_{\odot}}$ is the solar rotation frequency and $T_{\odot}=$ 25.3 days is the (equatorial) solar rotation period, and $A$ is the equatorial and $C$ the polar momentum of inertia. From observations one can find the form factor, $J_{2}=310^{-7}$ (Sofia et al. 1994), which is connected with the momenta of inertia by

$J_{2}=\frac{C-A}{M_{\odot} R_{\odot}} \approx \frac{C-A}{A}$

where $M_{\odot}$ is the Sun's mass and $R_{\odot}$ the solar equatorial radius. Inserting Eq. (3) in Eq. (2) and inverting gives the precession period of $\approx 2.310^{5}$ years. This period is far too large to explain the observed migration of the solar wind flow angle. Thus we can exclude precession effects as an explanation for the flow angle variations.

Planetary perturbations cause the Sun to move around the barycentre of the solar system. This motion is dominated by Jupiter and by Saturn and has a quasiperiodicity of $\approx 10$ years (Charvatova 1990). Because this motion does not change the rotation axis of the Sun, it can also not explain the drifts in the solar wind flow angles.

\subsection{Magnetic field effects}

Having excluded all gravitational effects, the causes for variations in the flow direction as well as changes of the angular momentum must be searched for in other reasons, for example in the regular changes occurring during the solar cycle in the global corona. The boundary conditions of the solar wind and the coronal plasma and magnetic field inside the Alfvén surface do change as a function of time. For the most recent results see the special issue of SOHO edited by Fleck \& Švestka (1997). At solar minimum the coronal hole boundaries in general remain at relatively high latitudes, $\approx 60^{\circ}$, but due to overexpansion the wind emanating from them reaches to much lower latitudes in interplanetary space. However, equatorial extensions of the coronal holes at specific longitudes do often reach into the equatorial regions. Especially during the declining phase of the solar cycle coronal holes reach deep into the equatorial regions, as was observed in 1974 by Skylab (Timothy et al. 1975) with the famous boot-shaped coronal hole; and more recently with the "elephants trunk" observed by SOHO in 1996 (Del Zanna \& Bromage 1999; Hoeksema et al. 1999) Therefore, during this period the solar wind speed is on average larger at low latitudes than 
during solar maximum periods. At solar maximum, coronal holes occur only rarely near the equator, and therefore mainly low-speed solar wind exists in the ecliptic. During solar maximum the Alfvén surface inside of which the solar wind partly corotates, is located at larger solar distances. Therefore, a larger transverse velocity component is imprinted on the wind flow. This may lead to a solar-cycle dependence on the variation of the transversal mechanical angular momentum of the solar wind.

From the theoretical point of view, one often describes the Sun's magnetic field by two different basic types of magnetic fields: a curl-free inner magnetic field, $B^{\mathrm{S}}$ (Hoeksema 1982, 1983; Bagenal \& Gibson 1991; Zhao \& Hoeksema 1995) (with the field lines ending on the socalled source-surface), and the outer magnetic field, e.g. the classic Parker spiral field $B^{\mathrm{P}}$ (Parker 1963), or more recently the Fisk field, $B^{\mathrm{F}}$ (Fisk 1996).

Both approaches are quite successful in describing the magnetic field properties in their respective regions: the inner field is a potential field and describes the magnetic field structure inside a spherical source surface with radius $R_{\mathrm{S}}$, which is located near $2.5-3 R_{\odot}$, while the outer fields are assumed to be valid beyond the distance $R_{\mathrm{A}}$, i.e. beyond the Alfvén surface. Both fields cannot be linked directly together, because the $\vartheta, \varphi$-components of the outer field do not vanish at the source surface, and the outer field must not be curl free. Therefore, we require an intermediate solar magnetic field to exist, which is consistent with the boundary conditions and can be continuously connected to the inner coronal magnetic field as well as to the outer interplanetary magnetic field.

To our knowledge, there is presently no coronal and heliospheric magnetic field model having the required properties. Yet, first global numerical MHD simulations (see, e.g., Linker et al. 1999; Bravo \& Stewart 1997; Mikić \& Linker 1995; Wang et al. 1993) are available. But they consume a lot of computer time and do not yet include a realistic treatment of the coronal thermodynamics or the acceleration of the solar wind (Marsch 1997, and references therein). Moreover, no magnetic field measurements are available for the suggested intermediate region. Thus, we can only qualitatively discuss the physics occurring possibly in the intermediate region.

The above proposed model has the advantage that it can explain the changes in the angular momentum as well as the anti-correlation between the elevation angles of the flow observed by the Helios 1 and Helios 2 spacecraft. During solar minimum the coronal holes reach deep down into the equatorial region, from where fast solar wind can emanate close to the solar surface. This radially-expanding fast solar wind has only a weak transverse component, which corresponds to a corotation distance of only a few solar radii. Hence the resulting angular momentum will remain small. During solar maximum, the solar activity in the equatorial streamer belt is high, and the coronal holes are pushed toward the poles. Then only slow solar wind emerges at the low-latitude region, which may be dominated by strong magnetic fields corotating out to the Alfvén surface (located at several solar radii). Hence the angular momentum transferred to the unsteady solar wind originating at the top of the streamers (Sheeley et al. 1997) will be comparatively large. This scenario explains the main variations of the transverse angular momentum of the solar wind.

During the 22-year solar cycle, the solar magnetic field shows strong variations in which the dipole component vanishes and changes the polarity. However, the multipole components still exist, and a magnetic field axis can be defined. On the other hand, the multipole expansion of the solar surface field is based on a current-free description and the assumption that the magnetic field at the source surface is purely radial. This magnetic field description cannot be extended to the interplanetary magnetic field, because this has a strongly developed current sheet. In interplanetary space, where the Helios spacecraft acquired their data, the heliospheric magnetic field axis may be defined by the current sheet and should be different from the magnetic field axis as described by the current-free inner coronal field model. At no time will the angular momentum associated with the heliospheric magnetic field vanish, but always contribute to the total angular momentum carried by the solar wind plasma and field. Hence, the migration of the magnetic pole as shown in Fig. 3 may not refer to the dipole axis of the Sun's field but to a more generally defined heliospheric magnetic field axis, which is determined at all times during the solar cycle. The coupling between the heliospheric magnetic field and the field on the source surface is unknown.

The solar magnetic field is produced by a dynamo, which has to be non-axial symmetric. Therefore it is very likely, that the magnetic axis precesses around the rotation axis. A precession of the magnetic axis will be reflected in a rotational variation of the inner coronal magnetic field. This modulation will be imprinted on the intermediate solar magnetic field and thus be transferred even to the heliospheric magnetic field. Such a process could explain the observed longitudinal and latitudinal variations of the solar wind flow direction and connect them with the solar cycle. In such a picture the magnetic pole of the Sun is migrating on timescales of some decades as can be seen in Fig. 3, like the Earth's pole does on much larger time scales of some ten to hundred thousand years.

From the Helios observations and the above discussion the following empirical model for the global solar magnetic field seems appropriate: an inner magnetic field, described essentially by the source surface fields (Hoeksema et al. 1982, 1983; Bagenal \& Gibson 1991; Zhao \& Hoeksema 1995), a heliospheric magnetic field of the Parker or Fisk type, and an intermediate field transforming gradually into the heliospheric field. The intermediate field is located in the region between the source surface radius $\left(3.5 R_{\odot}\right.$, which is also grossly coincident with the sonic point of the flow) and the Alfvén radius $R_{\mathrm{A}}$ (which may range between $\approx 10-30 R_{\odot}$ ). Such an empirical field configuration can explain the different observations between the two Helios spacecraft, because Helios 1 is on average in 
the northern magnetic hemisphere, while Helios 2 stays predominately in the southern hemisphere. This configuration is also indicated by the normal (out-of-ecliptic) component of the angular momentum, which is negative in the southern hemisphere, and positive in the northern hemisphere.

\section{Conclusion}

We have argued that the observed long-term variations in the flow direction of the solar wind plasma are best explained by a three-component magnetic field, including a new intermediate field component. The observed angular momentum variations may be caused by a migration of the solar magnetic pole.

The analyzed Helios data show that the local variation of the latitudinal and longitudinal components of the total angular momentum are of the same order. However, the latitudinal variations in different magnetic hemispheres have the opposite sign in the data presented here, which implies, that the corresponding angular momentum loss (integrated from the north to south directions) vanishes. Thus, only the azimuthal total angular momentum loss remains, which is induced by the solar rotation. Therefore, a more detailed theoretical analysis of the mechanical and field angular momenta is needed, because the Weber \& Davis (1967) model does not take into account the threedimensional nature of the solar wind outflow and total angular momentum transport.

The time lag between the sunspot number and the latitudinal flow direction of the wind as observed by Helios 1 can be explained by the late occurrence of the coronal holes near the equatorial region. Their appearance is delayed to about two years after solar minimum, and thus the flow direction of the wind will change with the same retardation.

A three-part model of the global solar heliospheric magnetic field is quite useful, because the physical processes in the three regions are different. Inside the source surface field a current free magnetic field approximation is suitable. Most probably, the fast solar wind originates in this region. In between the source surface and the Alfvén surface the magnetic field still dominates dynamically the flow and imprints by enforced partial corotation a nonradial component on the slow solar wind. Outside the Alvfén surface the kinetic solar wind energy exceeds that of the magnetic field, which from thereon is frozen in the solar wind. Furthermore, the magnetic field is dynamically unimportant in the outer heliosphere. In conclusion, it is very meaningful to have different approximations for the three field regions. Inside the source surface is the current free field, outside the Alfvén surface it can be described by the Parker spiral field or the Fisk field. The intermediate field regime needs further theoretical investigation.

\section{References}

Altschuler, M. D., \& Newkirk, G. 1969, Sol. Phys., 9, 131

Bagenal, F., \& Gibson, S. 1991, J. Geophys. Res., 96, 17663

Banaszkiewicz, M., Axford, W. I., \& McKenzie, J. F. 1998, A\&A, 337, 940

Brandt, J. C. 1966, ApJ, 144, 1221

Bravo, S., \& Stewart, G. A. 1997, ApJ, 489, 992

Burlaga, L. F. 1995, Interplanetary magnetohydrodynamics (Oxford University Press)

Charvatova, I. 1990, Bull. Astron. Inst. Czech., 41, 56

Del Zanna, G., \& Bromage, B. J. I. 1999, J. Geophys. Res., 104, 9753

Dessler, A. J. 1967, Rev. Geophys, 5, 1

Fisk, L. 1996, J. Geophys. Res., 101, 15547

Fleck, B., \& Švestka, Z. 1997, The first results from SOHO (Kluwer Academic Publishers)

Forsyth, R. J., Balogh, A., Horbury, T. S., et al. 1996, A\&A, 316,287

Gosling, J. T., Bame, S. J., Feldman, W. C., et al. 1997, Geophys. Res. Lett., 24, 309

Hoeksema, J. T., Wilcox, J. M., \& Scherrer, P. H. 1982, J. Geophys. Res., 87, 110331

Hoeksema, J. T., Wilcox, J. M., \& Scherrer, P. H. 1983, JGR, 88,9910

Marsch, E. 1997, Observations and models of the fast and slow solar wind, ESA-SP, 415, 7

Marsch, E., \& Richter, A. K. 1984, J. Geophys. Res., 89, 5386

Linker, J. A., Mikić, Z., Bisecker, D. A., et al. 1999, J. Geophys. Res., 104, 9809

Mikić, Z., \& Linker, J. A. 1995, Large-scale structure of the solar corona and inner heliosphere, in Solar Wind Conference VIII, Dana Point, CA June 1995, 104

Richardson, J. D., Belcher, J. W., Lazarus, A. J., et al. 1995, Non-radial flow in the solar wind, in Wind Conference VIII, Dana Point, CA June 1995, 479

Parker, E. N. 1963, Interplanetary Dynamical Processes (Interscience Publishers, New York)

Pizzo, V., Schwenn, R., Marsch, E., et al. 1983, ApJ, 271, 335

Schatten, K. H., Wilcox, J. M., \& Ness, N. F. 1969, Sol. Phys., 6,442

Scherer, K. 2000, J. Geophys. Res., 105, 10329

Scherer, K., Gangophadyay, P., Gruntman, M., Judge, \& Darrell, L. 1997, J. Geophys. Res., 102, 4653

Schwenn, R. 1990, Large-Scale Structure of the Interplanetary Medium, in ed. R. Schwenn, \& E. Marsch, Physics of the Inner Heliosphere I, 99

Sheeley, N. R. Jr., Wang, Y.-M., Hawley, S. H., et al. 1997, ApJ, 484, 472

Sofia, S., Heaps, W., \& Twigg, L. W. 1994, ApJ, 427, 1048

Timothy, A. F., Krieger, A. S., \& Vaiana, G. S. 1975, Sol. Phys., 42, 135

Wang, A.-H., Wu, S. T., Suess, S. T., \& Poletto, G. 1993, Sol. Phys., 147, 55

Weber, E. J., \& Davis, L. 1967, ApJ, 148, 217

Zhao, X., \& Hoeksema, J. T. 1995, J. Geophys. Res., 100, 19

Zhao, X., Hoeksema, J. T., \& Scherrer, P. H. 1999, J. Geophys. Res., 104, 9735 